\title{
LETTER
}

Lymphoma

\section{Safety and efficacy of the BRAF inhibitor dabrafenib in relapsed or refractory hairy cell leukemia: a pilot phase-2 clinical trial}

\author{
Enrico Tiacci $\left(^{1}{ }^{1} \cdot\right.$ Luca De Carolis $^{1} \cdot$ Edoardo Simonetti $^{1} \cdot$ Mara Merluzzi $^{1} \cdot$ Antonio Bennati $^{1}$. \\ Vincenzo Maria Perriello ${ }^{1}$ - Alessandra Pucciarini ${ }^{1}$ - Alessia Santi ${ }^{1}$ - Alessandra Venanzi ${ }^{1}$ - Valentina Pettirossi ${ }^{1}$.

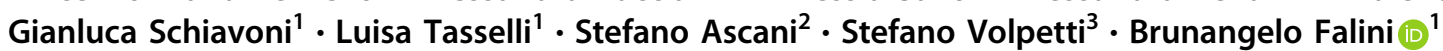

Received: 19 November 2020 / Revised: 25 January 2021 / Accepted: 24 February 2021 / Published online: 17 March 2021

(c) The Author(s) 2021. This article is published with open access

\section{To the Editor:}

Hairy-cell leukemia (HCL) is a rare indolent B-cell neoplasm that responds very well to chemotherapy with purine analogs (cladribine and pentostatin), but relapses are frequent (up to $58 \%$ in younger patients) $[1,2]$ and progressively less sensitive to these myelotoxic and immunesuppressive drugs. Our discovery that the $B R A F-\mathrm{V} 600 \mathrm{E}$ mutation is the genetic lesion underlying HCL [3-5] and shaping the morphologic, molecular and anti-apoptotic features of leukemic cells [6], opened the way to BRAF inhibition as targeted therapeutic strategy in this disease $[7,8]$.

Thus, we and others assessed the safety and efficacy of a short course of vemurafenib (an oral BRAF inhibitor clinically effective in BRAF-V600E + melanoma [9]) delivered to 50 evaluable relapsed/refractory HCL patients enrolled in two phase-2 clinical trials [10]. Vemurafenib (given for a median of 16 or 18 weeks) produced 96-100\% overall responses (ORs) and 35-42\% complete responses (CRs), all with measurable residual disease (MRD) [10]. In our trial with longer follow-up, the median survival free

Supplementary information The online version contains supplementary material available at https://doi.org/10.1038/s41375021-01210-8.

Enrico Tiacci

enrico.tiacci@unipg.it

$\bowtie$ Brunangelo Falini

brunangelo.falini@unipg.it

1 Hematology, University and Hospital of Perugia, Perugia, Italy

2 Anatomic Pathology, University of Perugia and Hospital of Terni, Perugia, Italy

3 Clinica Ematologica, Azienda Sanitaria Universitaria Integrata, Udine, Italy from relapse of cytopenias in the 25 patients reaching an OR was 9 months after the end of treatment [10].

Our pre-clinical studies on patients' HCL cells showed a promising anti-leukemic activity also for dabrafenib [6], another oral reversible ATP-competitive BRAF inhibitor approved in BRAF-V600E + metastatic melanoma [11]. Dabrafenib anti-leukemic activity was anecdotally reported also in single HCL patients, but response was not thoroughly documented [12] or disease burden was relatively limited [13] and did not clearly meet standard criteria for initiating treatment in HCL [14]. Therefore, we conducted a pilot phase-2 academic single-center clinical trial (EudraCT-2014001379-29) to prospectively assess dabrafenib safety and efficacy in $10 \mathrm{relapsed} / \mathrm{refractory}$ BRAF-V600E $+\mathrm{HCL}$ patients, including 2 who had been treated with vemurafenib within our previous trial [10].

Enrollment of the 10 patients (completed in 9 months) followed a Simon minimax statistical design aiming at an OR rate $\geq 60 \%$ (versus $<20 \%$ as null hypothesis) with $\alpha=0.05$ and $\beta=0.2$. Key inclusion criteria (see also Supplementary Appendix) were: (i) disease refractory to, or relapsing $\leq 2$ years after, the first course of purine analog, or relapsing whenever after a second or later course, or unsuitable to chemotherapy for patient comorbidites and/or old age; and (ii) disease requiring treatment for cytopenia(s) (neutrophils $<1500 / \mathrm{mm}^{3}$, platelets $<100,000 / \mathrm{mm}^{3}$ and/or hemoglobin $<11 \mathrm{~g} / \mathrm{dl}$ ).

Dabrafenib was given orally at its standard dose of $150 \mathrm{mg}$ twice daily for 8 weeks, followed by additional 4 weeks if no CR was obtained after 8 weeks. Response assessment, including splenic ultrasound and bone marrow (BM) evaluation, was performed every 4 weeks of dabrafenib dosing. Blood counts and chemistry were obtained weekly during the first 4 weeks, every 2 weeks thereafter during treatment, and every 3 months during follow-up. To monitor for dabrafenib toxicities, patients also underwent dermatologic examinations every 4 weeks and electrocardiograms every 2 weeks during treatment. 
As in our previous vemurafenib trial [10], CR required resolution of cytopenias (as defined above), no palpable splenomegaly and no leukemic hairy cells morphologically visible in the BM biopsy and peripheral blood smear. Partial remission (PR) required cytopenias resolution and $\geq 50 \%$ reduction of splenomegaly and HCL infiltration in the BM biopsy by immunohistochemistry. Minor response (MR) required an improvement of $\geq 50 \%$ in all abnormally low blood counts. Progression was defined as a HCL-related death, relapse or worsening of cytopenias after starting treatment, whichever occurred first.

The 10 patients (all males; median age: 62 years) had a median of 3.5 prior therapies, including a purine analog in all cases except one old patient previously treated with interferon (Table 1). Other previous treatments were interferon $(6 / 10$ patients, $60 \%)$, rituximab $(3 / 10,30 \%)$ as monotherapy $(n=2)$ or with pentostatin $(n=1)$, vemurafenib (2/10, 20\%), and splenectomy (2/10, 20\%). Median neutrophils were $455 / \mathrm{mm}^{3}$, platelets $56,000 / \mathrm{mm}^{3}$ and hemoglobin $11.1 \mathrm{~g} / \mathrm{dl}$. Median HCL infiltration in the BM biopsy was $80 \%$ (range $40-95 \%$ ). Five of the 8 (63\%) nonsplenectomized patients had splenomegaly (longest spleen diameter: $14.5-28 \mathrm{~cm}$ ).

All patients received dabrafenib for 12 weeks, except one reaching CR after 8 weeks. The OR rate was $80 \%$, including 3 CR $(30 \%)$ and 5 PR (50\%). Median time to recovery of platelets $\left(\geq 100,000 / \mathrm{mm}^{3}\right)$, neutrophils $\left(\geq 1500 / \mathrm{mm}^{3}\right)$ and hemoglobin $(\geq 11 \mathrm{~g} / \mathrm{dl})$ in responding patients was respectively 15 days (range: 6-36), 35 days (range: 11-58) and 51 days (range: 43-64).

The clinical benefit of dabrafenib lasted relatively long in the 3 complete responders, despite all had MRD ( 5-10\% HCL cells by immunohistochemistry in the BM biopsy post-therapy). One $\mathrm{CR}$ patient is progression-free at 60.5 months. The other two patients formally progressed at 15.5 and 14 months from starting treatment, but required further therapy only later, respectively 31 and 21 months after ending dabrafenib. Indeed, as with vemurafenib [10], blood counts indicative of relapse per protocol (e.g., neutrophils $<1500 / \mathrm{mm}^{3}$ ) can remain for a relatively long time above or around the thresholds commonly triggering antiHCL therapy in routine care (e.g., neutrophils $<1000 / \mathrm{mm}^{3}$ ) [14]. Furthermore, 1/3 CR patients had previously received a short vemurafenib treatment twice [10], which produced a CR in both instances but a shorter progression-free survival (PFS) with the second vemurafenib course (10.5 months) compared to the first one (16 months). Interestingly, PFS with dabrafenib was 14 months, comparing well with the last vemurafenib course.

Also 1/5 PR patients enjoyed a long survival free from progression (occurring at 42 months) and from a new therapy (started at 56 months), despite a previous splenectomy (which seems to associate with lower efficacy of vemurafenib
[10] and moxetumomamb pasudotox [15]). Conversely, in 4/5 PR patients, progression occurred 5.5-10 months after starting treatment and was followed by another therapy in 3 cases (10-13 months after ending dabrafenib) or death in 1 case (pneumonia 9 months after ending dabrafenib). The latter patient had previously achieved only PRs after being treated twice with vemurafenib.

The remaining $2 / 10$ patients, although reaching only MR, had significant clinical benefit from dabrafenib. In particular, one severely pancytopenic patient with huge splenomegaly $(28 \mathrm{~cm})$ and high transfusion requirement (4 units of erythrocytes and 4 of platelets per month), quickly became transfusion-independent (within 10 days from starting treatment) and did not eventually qualify for PR just because of residual mild thrombocytopenia $\left(70,000 / \mathrm{mm}^{3}\right)$. However, the beneficial effect of dabrafenib, including considerable reduction of splenomegaly $(17.5 \mathrm{~cm})$, allowed him to safely undergo a laparoscopic removal of the spleen, which would have been hardly feasible at baseline and which got rid of the main residual disease reservoir (as BM leukemic infiltration postdabrafenib was relatively mild). Indeed, splenectomy resulted in resolution of thrombocytopenia and freedom from relapse at 56 months. Also the other minor responder (75-year old) did not qualify for PR because of mild thrombocytopenia $\left(80,000 / \mathrm{mm}^{3}\right)$, and enjoyed a relatively long treatment-free survival (14.5 months).

Overall survival in the 10 patients was $90 \%$ at a median follow-up of 64 months from starting treatment (range: 14-79) (Fig. 1A).

Dabrafenib-related toxicities (Fig. 1B) were similar to those observed in melanoma trials [11] and were mostly of grade- $1 / 2$, none of grade- 4 and none leading to permanent drug discontinuation. They were all reversible and usually represented by arthralgias, facial flushing, warts, and asymptomatic increase of the QTc interval or of liver/pancreatic function tests. As with vemurafenib [10], no significant myelosuppression was observed. Four patients (40\%) received the full dabrafenib dose ( $150 \mathrm{mg}$ b.i.d.) for the whole planned duration, whereas in 6 patients $(60 \%)$ the dose was reduced (to $100 \mathrm{mg}$ b.i.d., $n=5$; to $50 \mathrm{mg}$ b.i.d., $n=1$ ) for a total of 29-56 days per patient, due to grade-2 arthralgia, grade-3 arthritis or asymptomatic laboratory abnormalities (grade-2/3 lipase increase; grade-3 phosphate decrease; grade-1 QTc prolongation). Most of these patients (4/6) were then able to re-escalate the dose to $150 \mathrm{mg}$ b.i.d. Thus, the median dose intensity actually delivered to the 10 patients (relative to the intended dose of $150 \mathrm{mg}$ b.i.d. for the planned duration of $8-12$ weeks) was high at $88 \%$ (range: $78-100 \%$ ).

The spectrum of dabrafenib toxicities in HCL patients was largely similar to vemurafenib. However, in an indirect comparison (and considering that vemurafenib was given 


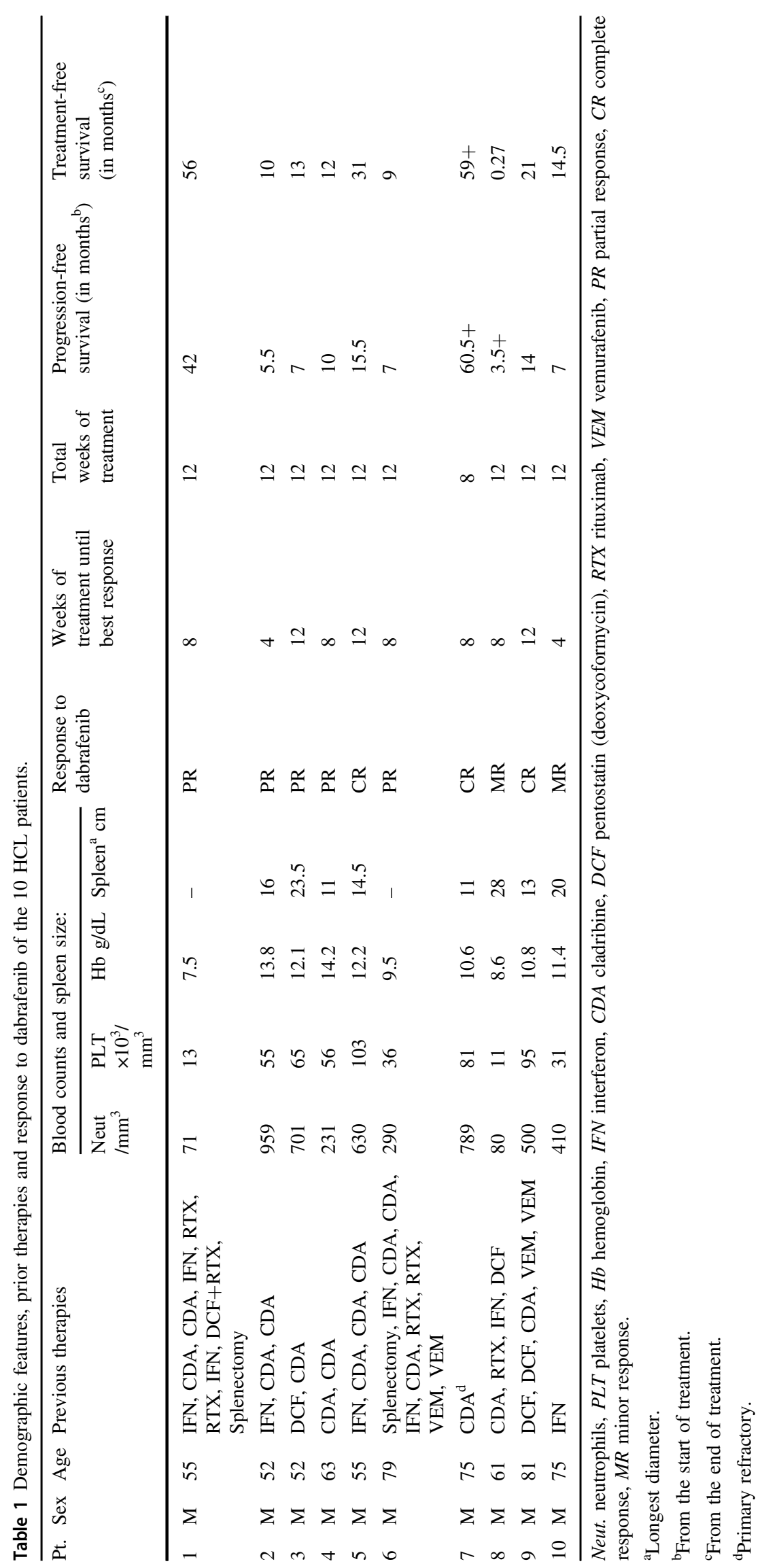


Fig. 1 Survival and toxicity after dabrafenib treatment in relapsed/refracotry HCL.

A Overall survival of the 10 patients treated with dabrafenib. B Toxicities of dabrafenib in the 10 patients.

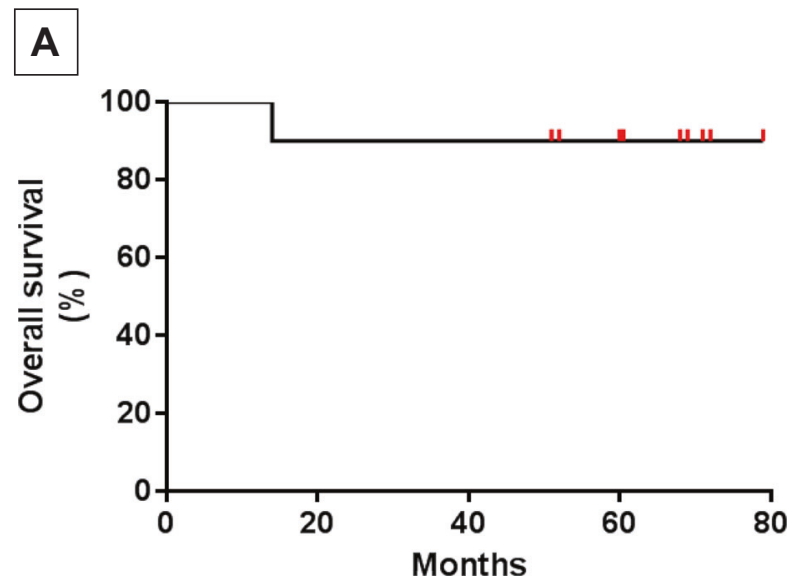

B

\begin{tabular}{|c|c|c|c|c|c|}
\hline \multicolumn{6}{|c|}{ Dabrafenib-related adverse events (graded according to CTCAEv4.03) } \\
\hline & Grade-1 & Grade-2 & Grade-3 & Grade-4 & Total \\
\hline Arthralgia/arthritis & $4(40)$ & $2(20)$ & $1(10)$ & 0 & $7(70)$ \\
\hline Rash or erythema & 0 & $1(10)$ & 0 & 0 & $1(10)$ \\
\hline Facial flushing & $5(50)$ & 0 & 0 & 0 & $5(50)$ \\
\hline Warts & $8(80)$ & 0 & 0 & 0 & $8(80)$ \\
\hline Hyperkeratosis & $1(10)$ & 0 & 0 & 0 & $1(10)$ \\
\hline QTe prolongation & $2(20)^{\#}$ & 0 & 0 & 0 & $2(20)$ \\
\hline Anemia ${ }^{\S}$ & $1(10)$ & $1(10)$ & 0 & 0 & $2(20)$ \\
\hline Hypophosphoremia $^{\dagger}$ & $2(20)$ & 0 & $4(40)$ & 0 & $6(60)$ \\
\hline ALT $_{\text {increase }}{ }^{\dagger}$ & $2(20)$ & 0 & 0 & 0 & $2(20)$ \\
\hline ALP increase $^{\dagger}$ & $1(10)$ & 0 & 0 & 0 & $1(10)$ \\
\hline GGT increase $^{\dagger}$ & $1(10)$ & 0 & $1(10)$ & 0 & $2(20)$ \\
\hline Hyperbilirubinemia $^{\dagger}$ & $3(30)$ & 0 & 0 & 0 & $3(30)$ \\
\hline Amilase increase ${ }^{\dagger}$ & $2(20)$ & 0 & 0 & 0 & $2(20)$ \\
\hline Lipase increase $^{\dagger}$ & $1(10)$ & $2(20)$ & $2(20)$ & 0 & $5(50)$ \\
\hline
\end{tabular}

for a median of 16-18 weeks [10], versus 12 weeks for dabrafenib), rash seemed to occur less frequently with dabrafenib (1/10 cases $10 \%$; grade- 2$)$ than with vemurafenib (29/54 cases, 54\%; grade- $2, n=22$ cases; grade-3, $n=7$ cases) ( $p$ value 0.015 by Fisher's exact test). Furthermore, no cutaneous neoplasms (a known complication of prolonged treatment with BRAF inhibitors [16]) occurred in dabrafenib-treated patients, versus 7/54 (13\%) patients treated with vemurafenib [10]. Moreover, only one dabrafenib-treated patient $(10 \%)$ developed symptomatic grade-3 toxicity (elbow arthritis), compared to $17 / 54$ (31\%) patients with vemurafenib [10]. Finally, the drug dose had to be reduced for most $(\geq 50 \%)$ of the planned treatment duration in $1 / 10(10 \%)$ patients treated with dabrafenib (due to grade-2 arthralgia), compared to $9 / 27$ (33\%) evaluable HCL patients in our vemurafenib trial [10].
In conclusion, a short treatment with dabrafenib safely and quickly induced objective responses and significant clinical benefit in all relapsed/refractory HCL patients enrolled in this pilot study, including a few previously treated with vemurafenib. The anti-leukemic activity of dabrafenib appears overall similar to vemurafenib [10], with a possibly milder toxicity profile. Thus, dabrafenib may represent a valid alternative to vemurafenib both in HCL patients naive to a BRAF inhibitor and in those previously intolerant to vemurafenib (as shown in melanoma patients) [17]. CR depth with vemurafenib [10] or dabrafenib in relapsed/refractory HCL is lower (no MRD-negativity) compared to the antiCD22 immunotoxin moxetumomab pasudotox (35\% MRDnegative CR rate) [15], although we cannot exclude that BRAF inhibitor activity could deepen with more prolonged dosing. However, a short treatment combining vemurafenib 
with rituximab seems to produce superior results in relapsed/ refractory HCL (61\% MRD-negative CR rate [18]), and studies assessing the safety and efficacy of dabrafenib plus anti-CD20 immunotherapy are warranted.

Acknowledgements This work was supported by an ERC-FP7-2013 Consolidator Grant to ET (no. 617471). Further support was received by AIRC (grant IG-14447 to ET; grant Metastasis/5-per-mille no. 21198 to BF), Leukemia and Lymphoma Society (Scholarship in Clinical Research 2030-14 to ET), Italian Ministry of Health (grant RF-2016-02362264 to ET), and the Hairy Cell Leukemia Foundation (grant 2015 to ET and BF). We thank Prof. Francesco Zaja (Udine and Trieste, Italy), Dr. Marco Ladetto (Alessandria, Italy), Dr. Agostino Antolino (Ragusa, Italy) and Dr. Giacinto La Verde (Rome, Italy) for contributing patient clinical informations.

\section{Compliance with ethical standards}

Conflict of interest ET and BF filed a patent on the use of mutant BRAF as HCL biomarker.

Publisher's note Springer Nature remains neutral with regard to jurisdictional claims in published maps and institutional affiliations.

Open Access This article is licensed under a Creative Commons Attribution 4.0 International License, which permits use, sharing, adaptation, distribution and reproduction in any medium or format, as long as you give appropriate credit to the original author(s) and the source, provide a link to the Creative Commons licence, and indicate if changes were made. The images or other third party material in this article are included in the article's Creative Commons licence, unless indicated otherwise in a credit line to the material. If material is not included in the article's Creative Commons licence and your intended use is not permitted by statutory regulation or exceeds the permitted use, you will need to obtain permission directly from the copyright holder. To view a copy of this licence, visit http://creativecommons. org/licenses/by/4.0/

\section{References}

1. Rosenberg JD, Burian C, Waalen J, Saven A. Clinical characteristics and long-term outcome of young hairy cell leukemia patients treated with cladribine: a single-institution series. Blood. 2014;123:177-83.

2. Else M, Dearden CE, Catovsky D. Long-term follow-up after purine analogue therapy in hairy cell leukaemia. Best Pract Res Clin Haematol. 2015;28:217-29.

3. Tiacci E, Trifonov V, Schiavoni G, Holmes A, Kern W, Martelli MP, et al. BRAF mutations in hairy-cell leukemia. N Engl J Med. 2011;364:2305-15.
4. Tiacci E, Schiavoni G, Forconi F, Santi A, Trentin L, Ambrosetti A, et al. Simple genetic diagnosis of hairy cell leukemia by sensitive detection of the BRAF-V600E mutation. Blood. 2012;119:192-5.

5. Tiacci E, Schiavoni G, Martelli MP, Boveri E, Pacini R, Tabarrini A, et al. Constant activation of the RAF-MEK-ERK pathway as a diagnostic and therapeutic target in hairy cell leukemia. Haematologica. 2013;98:635-9.

6. Pettirossi V, Santi A, Imperi E, Russo G, Pucciarini A, Bigerna B, et al. BRAF inhibitors reverse the unique molecular signature and phenotype of hairy cell leukemia and exert potent antileukemic activity. Blood. 2015;125:1207-16.

7. Falini B, Martelli MP, Tiacci E. BRAF V600E mutation in hairy cell leukemia: from bench to bedside. Blood. 2016;128:1918-27.

8. Tiacci E, Pettirossi V, Schiavoni G, Falini B. Genomics of hairy cell leukemia. J Clin Oncol. 2017;35:1002-10.

9. Chapman PB, Hauschild A, Robert C, Haanen JB, Ascierto P, Larkin $\mathrm{J}$, et al. Improved survival with vemurafenib in melanoma with BRAF V600E mutation. $\mathrm{N}$ Engl J Med. 2011;364:2507-16.

10. Tiacci E, Park JH, De Carolis L, Chung SS, Broccoli A, Scott S, et al. Targeting mutant BRAF in relapsed or refractory hairy-cell leukemia. N Engl J Med. 2015;373:1733-47.

11. Hauschild A, Grob JJ, Demidov LV, Jouary T, Gutzmer R, Millward M, et al. Dabrafenib in BRAF-mutated metastatic melanoma: a multicentre, open-label, phase 3 randomised controlled trial. Lancet. 2012;380:358-65.

12. Vergote V, Dierickx D, Janssens A, Verhoef G, Tousseyn T, Vandenberghe $\mathrm{P}$, et al. Rapid and complete hematological response of refractory hairy cell leukemia to the BRAF inhibitor dabrafenib. Ann Hematol. 2014;93:2087-9.

13. Blachly JS, Lozanski G, Lucas DM, Grever MR, Kendra K, Andritsos LA. Cotreatment of hairy cell leukemia and melanoma with the BRAF inhibitor dabrafenib. J Natl Compr Canc Netw. 2015;13:9-13.quiz 13.

14. Grever MR, Abdel-Wahab O, Andritsos LA, Banerji V, Barrientos J, Blachly JS, et al. Consensus guidelines for the diagnosis and management of patients with classic hairy cell leukemia. Blood. 2017;129:553-60.

15. Kreitman RJ, Dearden C, Zinzani PL, Delgado J, Karlin L, Robak $\mathrm{T}$, et al. Moxetumomab pasudotox in relapsed/refractory hairy cell leukemia. Leukemia. 2018;32:1768-77.

16. Boussemart L, Girault I, Malka-Mahieu H, Mateus C, Routier E, Rubington $\mathrm{M}$, et al. Secondary tumors arising in patients undergoing BRAF inhibitor therapy exhibit increased BRAF-CRAF heterodimerization. Cancer Res. 2016;76:1476-84.

17. Jeudy G, Dalac-Rat S, Bonniaud B, Hervieu A, Petrella T, Collet E, et al. Successful switch to dabrafenib after vemurafenib-induced toxic epidermal necrolysis. Br J Dermatol. 2015;172:1454-5.

18. Falini B, Tiacci E. New treatment options in hairy cell leukemia with focus on BRAF inhibitors. Hematol Oncol. 2019;37 (Suppl 1):30-37. 